U.S. DEPARTMENT OF COMMERCE

National Institute of Standards and Technology

\section{NISTIR 4353}

NEW NIST PUBLICATION October 1990

\section{National PDES Testbed}

\section{Report Series}

\section{NIST STEP} Working Form Programmer's Reference

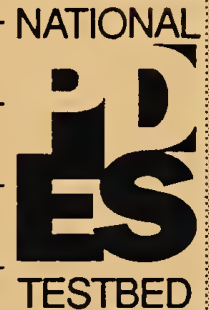





\section{NISTIR 4353}

National PDES Testbed

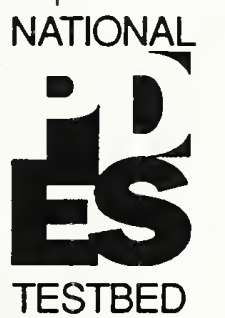

\begin{tabular}{l|}
\hline \\
\hline \\
\hline U.S. DEPARTMENT OF \\
\hline COMMERCE \\
\hline Robert A. Mosbacher, \\
\hline Secretary of Commerce \\
\hline
\end{tabular}

National Institute of

Standards and Technology

John W. Lyons, Director
NIST STEP Working Form Programmer's Reference

Stephen Nowland Clark

June 11, 1990 


\section{Disclaimer}

No approval or endorsement of any commercial product by the National Institute of

Standards and Technology is intended or implied

UNIX is a trademark of AT\&T Technologies, Inc. 


\section{Table Of Contents}

1 Introduction ...................................................................................

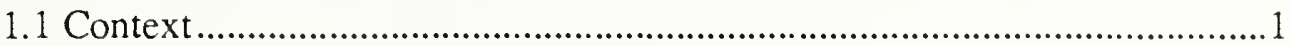

2 STEPparse Control Flow.........................................................2

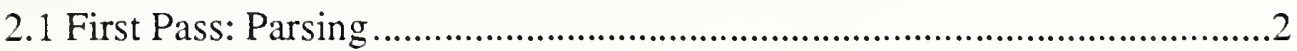

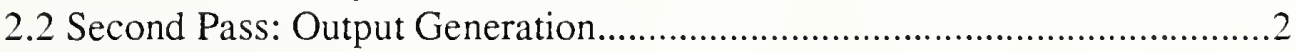

3 Working Form Implementation .....................................................3

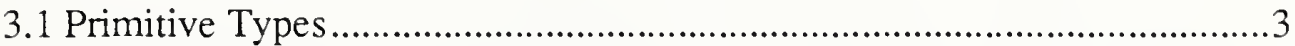

3.2 STEP Working Form Manager Module ...................................................

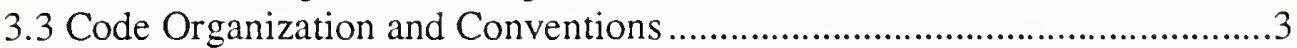

3.4 Memory Management and Garbage Collection ............................................4

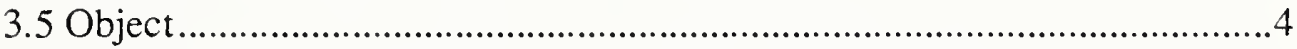

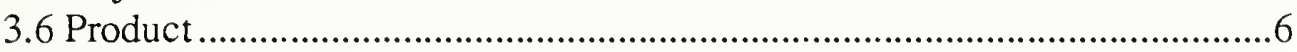

4 Writing An Output Module ......................................................7

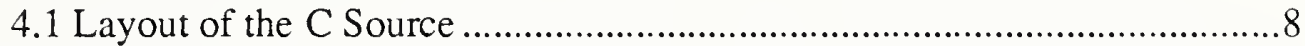

4.2 Output Module Linkage Mechanisms............................................................

5 Working Form Routines..........................................................9

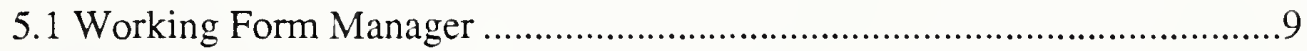

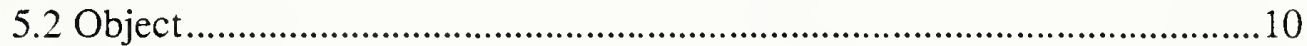

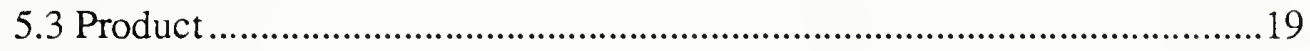

6 STEP Working Form Error Codes .............................................20

Appendix A: References .............................................................23 



\title{
NIST STEP Working Form Programmer's Reference
}

\author{
Stephen Nowland Clark
}

\section{Introduction}

The NIST STEP physical file parser [Clark90c], and its associated STEP parser, STEPparse, are Public Domain tools for manipulating product models stored in the STEP physical file format [Altemueller88]. These tools are a part of the NIST PDES Toolkit [Clark90a], and are geared particularly toward building STEP translators. This reference manual discusses the internals of the STEP Working Form, including STEPparse. The reader is assumed to be familiar with the design of the Toolkit ([Clark90a], [Clark90b], [Clark90c]). In some cases, technical knowledge of the Express Working Form [Clark90e] is also required.

The STEP Working Form relies on the NIST Express Working Form [Clark90b] as an in-core data dictionary, which provides a context in which STEP models can be interpreted. The tight dependency of the STEP Working Form abstractions on those of the Express Working Form is due to the schema-independent nature of the former. The STEP Working Form, and, in particular, STEPparse, contain no knowledge of any particular information model. Applications built on these tools can thus manipulate STEP product models in the context of any number of Express information models without requiring recompilation.

\section{$1.1 \quad$ Context}

The PDES (Product Data Exchange using STEP) activity is the United States' effort in support of the Standard for the Exchange of Product Model Data (STEP), an emerging international standard for the interchange of product data between various vendors' CAD/CAM systems and other manufacturing-related software [Smith88]. A National PDES Testbed has been established at the National Institute of Standards and Technology to provide testing and validation facilities for the emerging standard. The Testbed is funded by the CALS (Computer-aided Acquisition and Logistic Support) program of the Office of the Secretary of Defense. As part of the testing effort, NIST is charged with providing a software toolkit for manipulating PDES data. This NIST PDES Toolkit is an evolving, research-oriented set of software tools. This document is one of a set of reports which describe various aspects of the Toolkit. An overview of the Toolkit is provided in [Clark90a], along with references to the other documents in the set.

For further information on the STEP Working Form or other components of the Toolkit, or to obtain a copy of the software, use the attached order form. 
A STEPparse translator consists of two separate passes: parsing and output generation. The first pass builds an instantiated Product representing the product model specified in the STEP input file. This Product can then be traversed by an output module in the second pass, producing whatever report is desired. It is anticipated that users will need output formats other than those provided with the NIST Toolkit. The process of writing a report generator for a new output format is discussed in detail in section 4 .

\subsection{First Pass: Parsing}

The first pass of a STEPparse translator is a very simple parser. The STEPparse grammar itself is independent of any conceptual schema. The lexical analyzer recognizes any entity class name simply as an identifier; the actions associated with rules in the grammar then interpret this name as refering to a particular Express entity, and construct appropriate objects. As each construct is parsed, it is added to the Working Form. Because the STEP physical file format does not allow forward references to as-yet-undefined entity instances, all symbol references can be (and are) resolved during this parsing pass, so that no symbol resolution pass is required.

The STEPparse parser is written using the standard UNIX ${ }^{\mathrm{TM}}$ parser generation languages, Yacc and Lex. The grammar is processed by Bison, the Free Software Foundation' ${ }^{1}$ implementation of Yacc. The lexical analyzer is produced by Flex ${ }^{2}$, a fast, Public Domain implementation of Lex.

\subsection{Second Pass: Output Generation}

The report or output generation pass manages the production of the various output files. In the dynamically linked version of STEPparse, this pass loads successive output modules dynamically, calling each to traverse the Working Form. The dynamic linking mechanism is discussed briefly in [Clark90d]. It is also possible to build a statically linked translator, with a particular output module loaded in at build time; this is, in fact, the only mechanism available in an environment which is not derived from BSD 4.2 UNIX.

A report generator is an object module, most likely written in $\mathrm{C}$, which has been compiled as a component module for a larger program (i.e., with the - $c$ option to a Unix $C$ compiler). In the dynamically linked version, the object module is linked into the running parser, and its entry point (by convention a function called print_file ()) is

\footnotetext{
1. The Free Software Foundation (FSF) of Cambridge, Massachusetts is responsible for the GNU Project, whose ultimate goal is to provide a free implementation of the UNIX operating system and environment. These tools are not in the Public Domain: FSF retains ownership and copyright priviledges, but grants free distribution rights under certain terms. At this writing, further information is available by electronic mail on the Internct from gnu@prep.ai.mit.edu.

2. Vern Paxson's Fast Lex is usually distributed with GNU software, although, being in the Public Domain, it is not an FSF product and does not come under the FSF licensing restrictions.
} 
called. The code of this module consists of calls to STEP Working Form access functions and to standard output routines. Chapter 4 provides a detailed description of the creation of a new output module.

\section{Working Form Implementation}

As in the Express Working Form [Clark90d], the Object abstraction is implemented as a Symbol header block with a pointer to a private st ruct Object. This $\mathrm{C}$ structure contains the real definition of the abstraction, but is never manipulated directly outside of the Object module. Product is implemented as a pointer to a private structure, struct Product.

Most stylistic and other conventions from the Express Working Form are equally valid for STEP; they are reiterated here for emphasis.

3.1 Primitive Types

The STEP Working Form makes use of several modules from the Toolkit general libraries, including the Error and Linked_List modules. These are described in [Clark90d].

\subsection{STEP Working Form Manager Module}

In addition to the abstractions discussed in [Clark90c], I ibstep. a contains one more (conceptual) module, the package manager. Defined in step.c and step.h, this module includes calls to intialize the entire STEP (and Express) Working Form package, and to run each of the passes of a STEPparse translator.

\subsection{Code Organization and Conventions}

Each abstraction is implemented as a separate module. Modules share only their interface specifications with other modules. A module Foo is composed of two $\mathrm{C}$ source files, foo.c and foo.h. The former contains the body of the module, including all non-inlined functions. The latter contains function prototypes for the module, as well as all type and macro definitions. In addition, global variables are defined here, using a mechanism which allows the same declarations to be used both for extern declarations in other modules and the actual storage definition in the declaring module. These globals can also be given constant initializers. Finally, foo. $\mathrm{h}$ contains inline function definitions. In a compiler which supports inline functions, these are declared stat ic inl ine in every module which includes foo. $h$, including foo.c itself. In other compilers, they are undefined except when included in $\mathrm{f} 00 . \mathrm{c}$, when they are compiled as ordinary functions. foo.c resides in $\sim$ pdes/src/step/; foo. $\mathrm{h}$ in $\sim$ pdes/include/.

The type defined by module Foo is named $\mathrm{F} \circ \mathrm{O}$, and its private structure is struct FOO. Access functions are named as FOO function (); this function prefix is abbreviated for longer abstraction names, so that access functions for type EOolhardy_Bartender might be of the form FOO_BARfunction(). Some 
functions may be implemented as macros; these macros are not distinguished typographically from other functions, and are guaranteed not to have unpleasant side effects like evaluating arguments more than once. These macros are thus virtually indistinguishable from functions. Functions which are intended for internal use only are named EOO_function (), and are usually static as well, unless this is not possible. Global variables are often named $\mathrm{FOO}$ variable; most enumeration identifiers and constants are named EOO_CONSTANT (although these latter two rules are by no means universal).

Every abstraction defines a constant $F O 0$ NULL, which represents an empty or missing value of the type. In addition, there are several operations which are defined for every type; these are primarily general management operations. Each abstraction defines at least one creation function, e.g. EOocreate (). The parameters to this creation function vary, depending on the abstraction. A permanent copy of an object (as opposed to a temporary copy which will immediately be read and discarded) can be obtained by calling EOOcopy (foo). This helps the system keep track of references to an object, ensuring that it is not prematurely garbage-collected. Similarly, when an object or a copy is no longer needed, it should be released by calling FOOfree ( $f \circ 0)$, allowing it to be garbage-collected if appropriate.

For each abstraction, there is a function FOOis_foo $\left(O b_{j}\right)$ which returns true if and only if its argument is a foo. This is useful when dealing with a heterogeneous list, for example.

\subsection{Memory Management and Garbage Collection}

In reading various portions of the STEP Working Form documentation, one may get the impression that the Working Form does some reasonably intelligent memory management. This is not true. The NIST PDES Toolkit is primarily a research tool. This is especially true of the Express and STEP Working Forms. The Working forms allocate huge chunks of memory without batting an eye, and this memory often is not released until an application exits. Hooks for doing memory management do exist (e.g., XXXfree () and reference counts), but currently are largely ignored.

\subsection{Object}

The Object abstraction is the basic building block of the STEP Working Form. An Ob ject is created for each unit of value in a PDES/STEP product model: each entity instance, aggregate, integer, string, etc. On the surface, this would seem to be a reasonably straightforward module to implement: each ob ject has an optional name, a Type, and a value. The value may be simple or structured; in either case, it basically comes down to a pointer - either to an array of objects, or to an integer, real, string, etc.

As with most abstractions in the Express Working Form, object is implemented as a Symbol header whose definition field points at a struct object, which is defined thus:

$$
\text { struct object } 1
$$




Type type;
Generic user_data;
union \{
Constant enumeration;
Integer integer;
Logical logical;
Real real;
String string;
Object* entity;
Aggregate aggregate;
value;

The first two fields are pretty straightforward. Note that user_data is a generic pointer field. In strict ANSI C, only a pointer can be safely stored into this field and later retrieved; it is safest to only store pointers in this field. In particular, the age-old trick of casting pointers and integers back and forth, never completely portable, is now officially frowned upon.

The value union is where things get tricky. This field contains the actual value of the object represented. Unstructured types (numbers, logicals, and strings) are represented directly; e.g., object.value. integer contains an integer, and object.value.string, a character pointer. The value of an enumeration object is represented as a Constant, which will be an element of the appropriate enumeration. The integer representation of this enumeration element can be retrieved by calling (int)CSTget_value (object.value.enumeration).

An entity instance's value field, value. entity, is a pointer to the base of an array of objects. Each element of this array corresponds to an attribute of the entity; attributes appear in the same order as in a PDES/STEP physical file, with empty attributes explicitly represented by OBJECT_NULL. The offset to a particular attribute value is retrieved from the Express data dictionary by calling

ENTITYget_attribute_offset(entity, attribute), where entity is the entity class of the object in question and attribute is the Variable representing the attribute to be located.

The most convoluted object value representation is that for aggregates. An aggregate value is represented as a pointer to a struct Aggregate, defined as

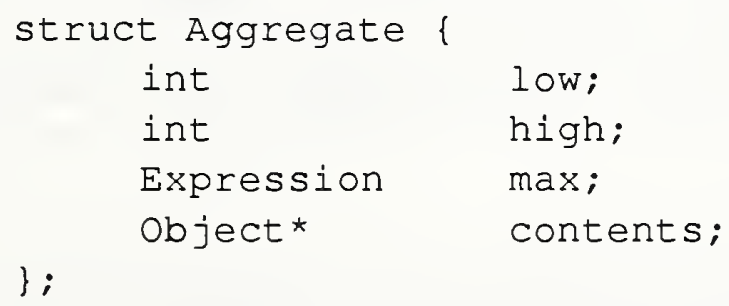

The last field, contents, holds the actual contents of the aggregate, as an array of objects. The low field provides a lower bound on allowable indices into this array, and doubles as a logical offset to the first element of the array. This value is 1 for any 
non-array aggregate. Thus, when low is 1, some aggregate [1] is found at contents [0]. Similarly, in an array whose low is 10, the some_array [12] is found at contents $[12-10=2]$. low remains constant in any particular aggregate object. The high field gives an upper bound on the indices of currently filled slots in an aggregate object. Every index into the aggregate beyond $\mathrm{h} i \mathrm{gh}$ which is in bounds is guaranteed to return OBJECT_NULL. The end result is that a loop of the form for ( $i=$ low; $i<=$ high; $++i$ ) <use contents $[i-l o w]>$ will always hit all of the elements of an aggregate. This function of offsetting by the lower bound is bundled into the various aggregate indexing functions of the working form (OBJaggr_at (), OBJlist_insert (), etc.), so that the indices which a user sees will be the ones which would be expected based on the Express model. In the current implementation, high in an aggregate whose type (from Express) gives a finite upper bound always remains constant at this bound. In the case of an aggregate with no specified upper bound, however, high may vary with the number of elements actually in the aggregate. The expression (from Express) giving the absolute upper bound on an aggregate is cached in aggregate->max. high is never allowed to be greater than the value of this expression.

The two calls OBJaggr_at () and OBJaggr_at_put () can be used with any kind of aggregate, although they are intended to be used primarily for building general aggregates which will later be OBJtype_cast () into specific types of aggregates.

This is how STEPparse builds aggregates, since it is considerably easier than figuring out at parse time what type of aggregate should be built. The various class-specific manipulations (list concatenation, set intersection, etc.) are provided by calls requiring aggregates of a particular class: OBJlist_concat (), OBJset_intersect (), etc. It should be noted that the calls for combning aggregates are destructive: each modifies its first argument to hold its computed result. In general, the two arguments may safely be set equal. Exceptions are noted in the individual function specifications.

Finally, a word about type conversion (also known as casting, as in C). Type conversions of existing Objects are handled by OBJtype_cast (Object, Type, Error*). Only certain conversions are allowed; other attempted casts leave the Object unchanged and return an error code. Clearly, any Object can trivially be cast into its own type. The different numeric types can be cast about at will. A general aggregate can be cast into any specific aggregate class; otherwise, an aggregate can only be cast into another aggregate type of the same class: an array cannot be cast into a set, etc. Each element of the aggregate being cast must, of course, be recursively cast into the appropriate base type; each of these conversions is subject to the same rules as any other cast. Finally, an entity Ob ject can be converted into an instance of a supertype of its class, or into an instance of a SELECT type containing some type to which it can be cast. These casts of entity instances in fact do not modify the object being cast.

\subsection{Product}

A product in STEP contains a large number of interrelated entity instances, and is represented by the Product abstraction. Each Product is named, and includes a pointer to the Express model which provides the scope in which its component Ob jects are 
defined. These component objects can be retrieved from the Product in several ways: a specific (external) entity instance can be retrieved by name; a Linked_List of all of the (external) entity instances in the Product can be requested; or a particular entity class in the Product's conceptual schema can be queried for all of its instances (note that this last method retrieves both internal and external entity instances). Internal (embedded) entity instances and non-entity Objects must appear as attribute values or aggregate elements somewhere in the Product, and are only accessible via ENTITYget_instances () and component retrieval from the containing Objects.

The above three access methods are supported by storing three references to each object in a Product. When an Object is added to a Product, it is added to the end of the list of external objects. This list preserves the order in which the objects were added to the Product, and so is appropriate for applications, such as writing a STEP physical file, which require that there be no forward references to as-yet-undefined Objects. Each external Object is also added to a dictionary which the Product maintains, to allow retrieval by name. And when an entity object is first created, it is added to the instance list of its class.

\section{$4 \quad$ Writing An Output Module}

We now turn to the topic of actually writing a report generator. The end result of this process will be an object module (UNIX . $\circ$ file) which can be loaded into STEPparse. This module contains a single entry point which traverses a given Product and writes its output to a particular file. The conceptual entry point is conventionally called print_file(), while the physical entry point, which simply dispatches to print_file(), is called entry_point ().

In most cases, there will be a one-to-one correspondence between Objects in the instantiated Working Form and records to be written on the output. When this is the case, the meat of the report generator can be made fairly simple. Since a list of all of the Objects in the Working Form is available, it is easy to iterate over this list and output each Object in sequence:

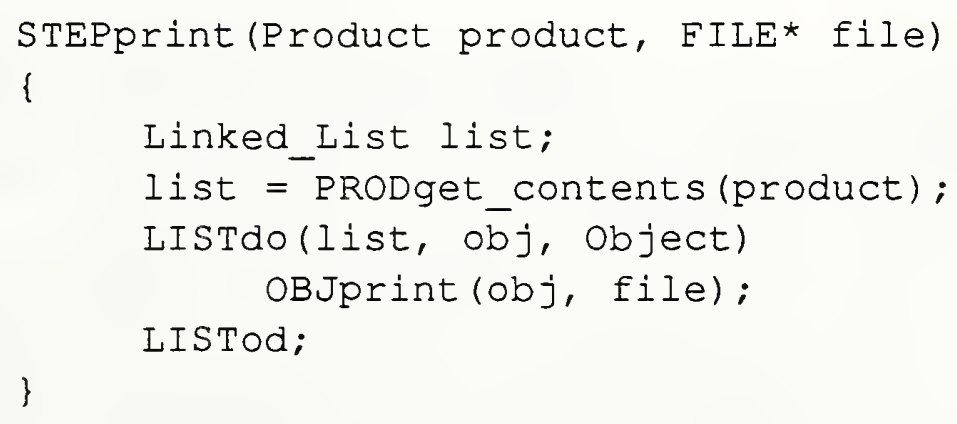

The only remaining problem is to write a function OBJprint () which emits the output record for a single Object. Given the variety of types of Objects, this function will probably be controlled by a large switch statement, selecting on the Object's type class (numbers, strings, and aggregates all have to be printed differently). Code to deal with multi-dimensional arrays an internal/external entity references can get tricky, and 
should be written carefully. An example of a fairly simple report generator is that used by STEPparse-QDES. The source code for this module is in $\sim$ pdes/src/STEPparse_qdes/step_output_smalltalk.c.

\subsection{Layout of the C Source}

The layout of the $\mathrm{C}$ source file for a report generator which will be dynamically loaded is of critical importance, due to the primitive level at which the load is carried out. The very first piece of $C$ source in the file must be the entry_point () function, or the loader may find the wrong entry point to the file, resulting in mayhem. Only comments may precede this function; even an \# include directive may throw off the loader. An output module is normally layed out as shown:

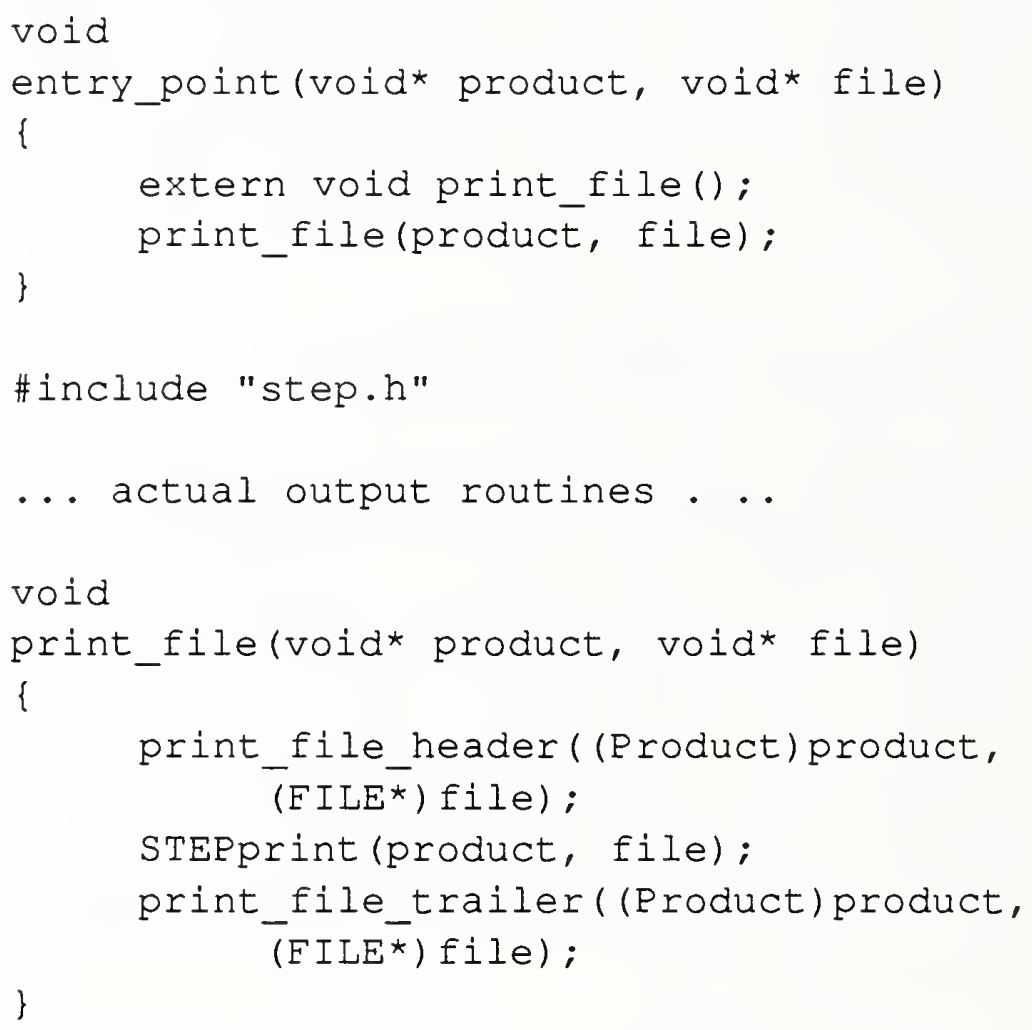

The print_file () function will probably always be quite similar to the one shown, although in many cases, the file header and/or trailer may well be empty, eliminating the need for these calls. In this case, STEPprint () and print_file () will probably become interchangeable.

Having said all of the above about templates, code layout, and so forth, we add the following note: In the final analysis, the output module really is a free-form piece of $\mathrm{C}$ code. There is one and only one rule which must be followed: The entry point (according to the $a$.out format) to the .0 file which is produced when the report generator is compiled must be appropriate to be called with a Product and a FILE*. The simplest (and safest) way of doing this is to adhere strictly to the layout given, and write an entry_point () routine which jumps to the real (conceptual) entry point. But any other convention which guarantees this property may be used. 


\subsection{Output Module Linkage Mechanisms}

One of the powers of STEPparse is the flexibility which it gives a user with regard to generating output. An important component of this flexibility on BSD Unix systems is the dynamic loading of output modules. Both static and dynamic binding of output modules are supported by STEPparse. This is implemented by physically breaking the object code from the Working Form manager (step.c) into three separate . $\circ$ files: the initialization code and the first pass of STEPparse are compiled into step. $\circ$, which is stored in libstep. a. The static linking version of the second pass (without any output module) is compiled into step_static. 0 ; and the dynamic loading version into step_dynamic. o. Sources for all of these components reside in step.c; the various sections are extracted via conditional compilation: When this file is compiled with the preprocessor symbols reports and static_reports defined, step_static.o is produced. With reports and dynamic_reports defined, step_dynamic. $\circ$ is produced; and with none of these defined, step. $\circ$ is produced.

Since step_static.o and step_dynamic.o both define the function STEPreport (), only one can be linked into any given executable. This selection is what determines whether a STEPparse translator links in output modules statically or dynamically. Note that a suitable output module (.$\circ$ file) must appear after step_static.o in the linker's argument list when a statically linked translator is being built.

\section{$5 \quad$ Working Form Routines}

The remainder of this manual consists of specifications and brief descriptions of the access routines and associated error codes for the STEP Working Form. The error codes are manipulated by the Error module [Clark90d]. Each subsection below corresponds to a module in the Working Form library. The Working Form Manager module is listed first, followed by the remaining data abstractions in alphabetical order.

\section{$5.1 \quad$ Working Form Manager}

\begin{tabular}{|c|c|}
\hline Procedure: & STEPinitialize \\
\hline Parameters: & Error* errc - buffer for error code \\
\hline Returns: & void \\
\hline Description: & $\begin{array}{l}\text { Initialize the STEP Working From package. In a typical STEP translator, this is call } \\
\text { by the default ma in () provided in the Working Form library. Other applications } \\
\text { should call this function at initialization time. }\end{array}$ \\
\hline Errors: & none \\
\hline Procedure: & STEPparse \\
\hline \multirow[t]{2}{*}{ Parameters: } & String filename - the name of the file to be parsed \\
\hline & Express data_model - conceptual schema (as produced by EXPRESSpass $2(1)$ ) \\
\hline Retu & Product - the product model parsed \\
\hline Description: & Parse a STEP physical file into the Working Form \\
\hline
\end{tabular}


Procedure:

Parameters:

Returns:

Description:

Description:
STEPreport

Product product - the product to output

void

Invoke one or more report generators for a STEP Working Form model.

Invoke one (or more) report generator(s). When this function is compiled with -Ddynamic_reports, it will repeatedly prompt for report generators and output files, dynamically loading and executing them. When it is compiled with

-Dstatic_reports, a report generator must also be included at link time, with the entry point print_file(Express, FILE*).

\section{$5.2 \quad$ Object}

Procedure: OBJaggr_at

Parameters: Object object - object to examine

int index - index of requested element

Error* errc - buffer for error code

\section{Returns: $\quad$ Object - value at requested position}

Description: Retrieves the value at some position in an aggregate. Note that the calls which are specific to a particular aggregate class are much to be preferred.

Errors: ERROR_index_out_of_range - the index is outside of the bounds of the aggregate

Procedure: OBJaggr_at_put

Parameters: Object object - object to modify

int index - index at which to put element

Object value - value to insert

Error* errc - buffer for error code

Returns:

void

Description: Store a value into an aggregate object. Note that the calls which are specific to a particular aggregate class are much to be preferred.

Errors: ERROR_index_out_of_range - the index is outside of the bounds of the aggregate

Procedure: OBJaggr_lower_bound

Parameters: Object object - object to examine

Error* errc - buffer for error code

Returns: int - the lower bound of the object

Description: Retrieves the lower bound of an aggregate object. For an array, this is the index of the

Errors: first element of the array. For other aggregates, it is 1.

Procedure: OBJaggr_upper_bound

Parameters: Object object - object to examine

Error* erre - buffer for error code

Returns: int - the upper bound of the object

Description: Retrieves the upper bound of an aggregate object. For an aggregate with a constrained size, this is the value of the upper limit or index. For an aggregate with an infinite upper bound, the value returned is guaranteed to be larger than the highest index of a filled slot in the aggregate.

Errors: none 


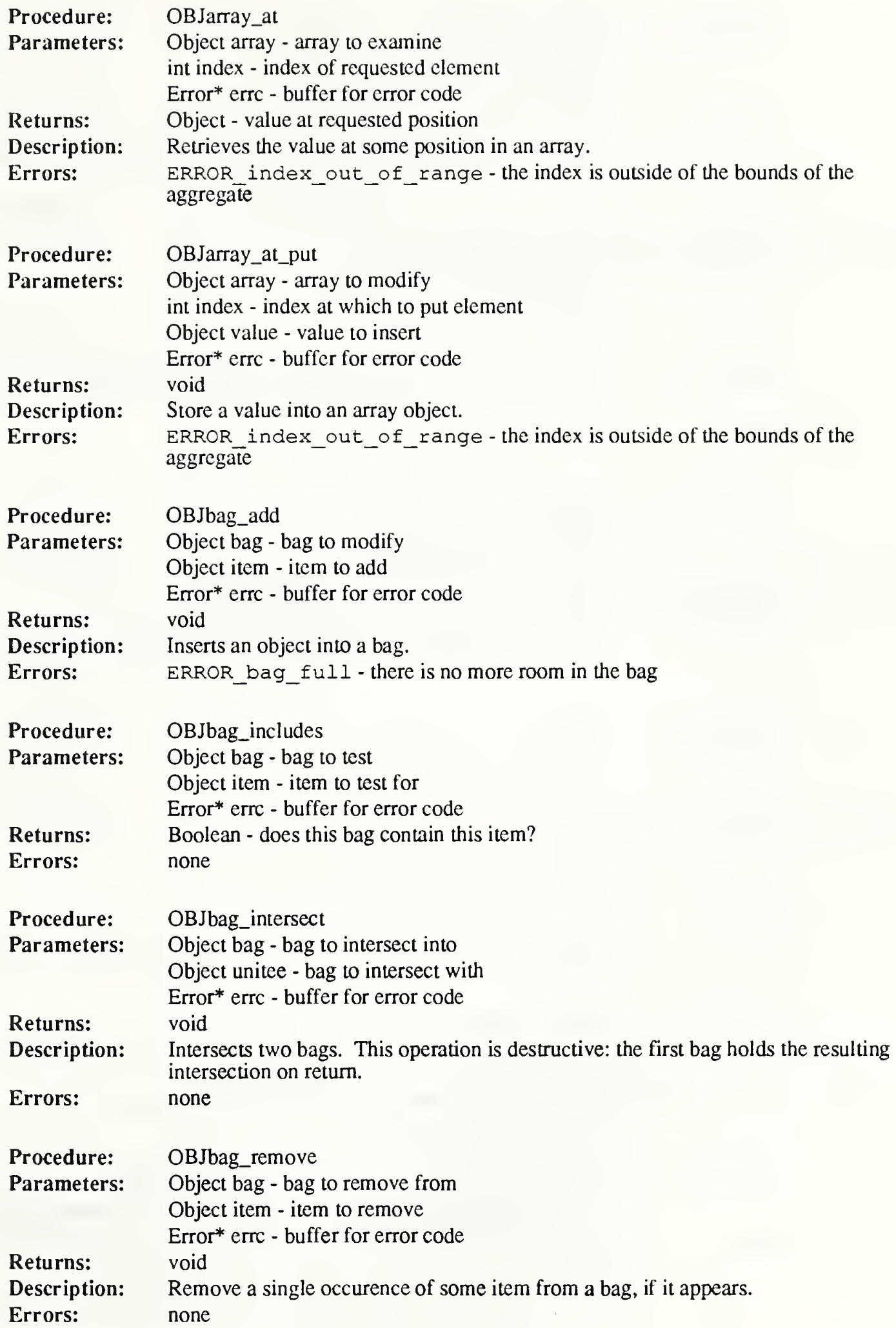

Errors: none

Procedure: OBJbag_remove

Parameters: Object bag - bag to remove from

Object item - item to remove

Error* errc - buffer for error code

Returns: void

Description: Remove a single occurence of some item from a bag, if it appears.

Errors: none 


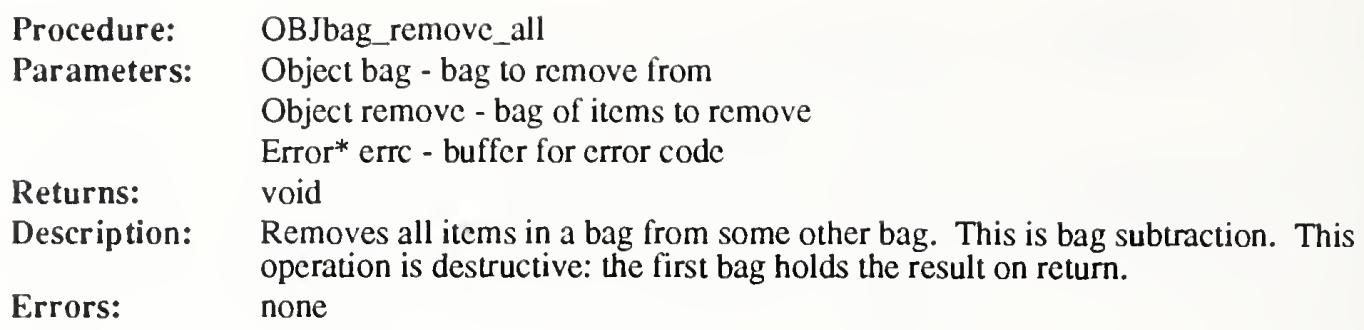

Procedure: OBJbag_subset

Parameters: Object bag - bag to test as superset

Object subset - bag to test as subset

Error* errc - buffer for crror code

Returns: Boolean - does the first bag contain the second as a subset?

Description: This implementation is not completely correct. In particular, the following returns

Errors: none

Procedure: OBJbag_unite

Parameters: Object bag - bag to unite onto

Object unitee - bag to unite with

Error* errc - buffer for error code

Returns: $\quad$ void

Description: Adds the contents of a bag to another bag. This operation is destructive: the first bag

Errors: none

Procedure: OBJcopy

Parameters: Object object - object to copy

Returns: $\quad$ Object - a shallow copy of the object

Procedure: OBJcreate

Parameters: Type type - type to instantiate

Error* errc - buffer for error code

Returns: $\quad$ Object - a new, empty object of the given type

Errors: $\quad$ ERROR_cannot_instantiate - the type given cannot be instantiated (e.g., Generíc)

Procedure: OBJcreate_entity

Parameters: Entity entity - entity class to instantiate

Linked_List attributes - list of attribute values

int line - source line number of the instance to be created

Error* errc - buffer for error code

Returns: $\quad$ Object - a new entity instance, as described

Description: A new object of the specified entity type is created. There should be a one-to-one correspondence between the values on the attribute value list and the list of attributes for the entity being instantiated.

Errors: $\quad$ ERROR insufficient_attributes - not enough attribute values in the list provided

ERROR_too_many_attributes - too many attribute values in the list provided 
Procedure: OBJcreate_ud_entity

Description: Creatc a user-defined entity. This procedure is not yet implemented.

Procedure: OBJfast_get_attribute

Parameters: Object object - object to examine

Variable attribute - attribute to retrieve

Error* errc - buffer for error code

Returns: Object - value of attribute

Description: Retrieves the value of an attribute from an entity instance. This call is faster than OBJget_attribute () when the caller already has the actual attribute record for the desired attribute, rather than simply having its name (as expected by OBJget_attribute()).

Errors: none

Procedure: OBJfast_put_attribute

Parameters: Object object - object to modify

Variable attribute - attribute to store into

Object value - value to store into attribute

Error* errc - buffer for error code

Returns: void

Requires: TYPEget_class(OBJget_type $(o b j e c t))==$ TYPE_ENTITY

Description: Store a value into an attribute of an entity instance. This call is faster than OBJput attribute () when the caller already has the actual attribute record for the desired attribute, rather than simply having its name (as expected by OBJput_attribute()).

Errors: Same as for OBJput_attribute().

Procedure: OBJfree

Parameters: Object object - object to free

Error* errc - buffer for error code

Returns: void

Description: Release an Object. Indicates that the object is no longer used by the caller; if there are no other references to the object, all storage associated with it may be released.

Errors: none

Procedure: OBJget_attribute

Parameters: Object object - object to examine

String attributeName - name of attribute to retrieve

Error* errc - buffer for error code

Returns: Object - value of the named attribute

Description: Retrieves the value of a named attribute from an entity instance. This call is the slower method for retrieving an attribute value. If the actual attribute recored is already available, use OBJfast_get_attribute () instead.

Errors: none

Procedure: OBJget_line_number

Parameters: Object object - object to examine

Returns: int - line number of object

Errors: none 


\begin{tabular}{|c|c|}
\hline Procedure: & OBJget_name \\
\hline Parameters: & Object object - object to examine \\
\hline Returns: & String - the object's name \\
\hline Description: & $\begin{array}{l}\text { Retrieves the name of an object. Unnamed objects, which would normaliy be } \\
\text { embedded entities and non-entities, yicld STRING NULL. }\end{array}$ \\
\hline Errors: & none \\
\hline Procedure: & OBJget_type \\
\hline Parameters: & Object object - object to examine \\
\hline Returns: & Type - the type of the object \\
\hline Errors: & none \\
\hline Procedure: & OBJget_user_data \\
\hline Parameters: & Object object - object to examine \\
\hline & Error* errc - buffer for error code \\
\hline Returns: & Generic - value of user data field for this object \\
\hline Errors: & none \\
\hline Procedure: & OBJget_value \\
\hline Parameters: & Object object - object to examine \\
\hline & Error* errc - buffer for error code \\
\hline Returns: & Generic - the object's value \\
\hline Description: & $\begin{array}{l}\text { Retrieves the value of a single-valued object. The value returned will be a char* for } \\
\text { a string object, a Constant for an enumeration object, and a pointer to an int, } \\
\text { double, or Boolean for an integer, real, or logical object, respectively. See } \\
\text { OBJarray_at (), OBJbag_includes (), OBJlist_at (), and } \\
\text { OBJset at () to read from an aggregate. See OBJget_attribute () to read } \\
\text { from an entity instance. }\end{array}$ \\
\hline Errors: & none \\
\hline Procedure: & OBJinitialize \\
\hline Parameters: & Error* errc - buffer for error code \\
\hline Returns: & void \\
\hline $\begin{array}{l}\text { Description: } \\
\text { Errors: }\end{array}$ & $\begin{array}{l}\text { Initialize the Object module. This is called by STEPinitialize } 0 \text {. } \\
\text { none }\end{array}$ \\
\hline Procedure: & OBJis_extemal \\
\hline Parameters: & Object object - object to examine \\
\hline Returns: & Boolean - is this an extemal object (non-embedded entity)? \\
\hline Errors: & none \\
\hline Procedure: & OBJis_internal \\
\hline Parameters: & Object object - object to examine \\
\hline Returns: & Boolean - is this an internal object (embedded entity)? \\
\hline Errors: & none \\
\hline
\end{tabular}




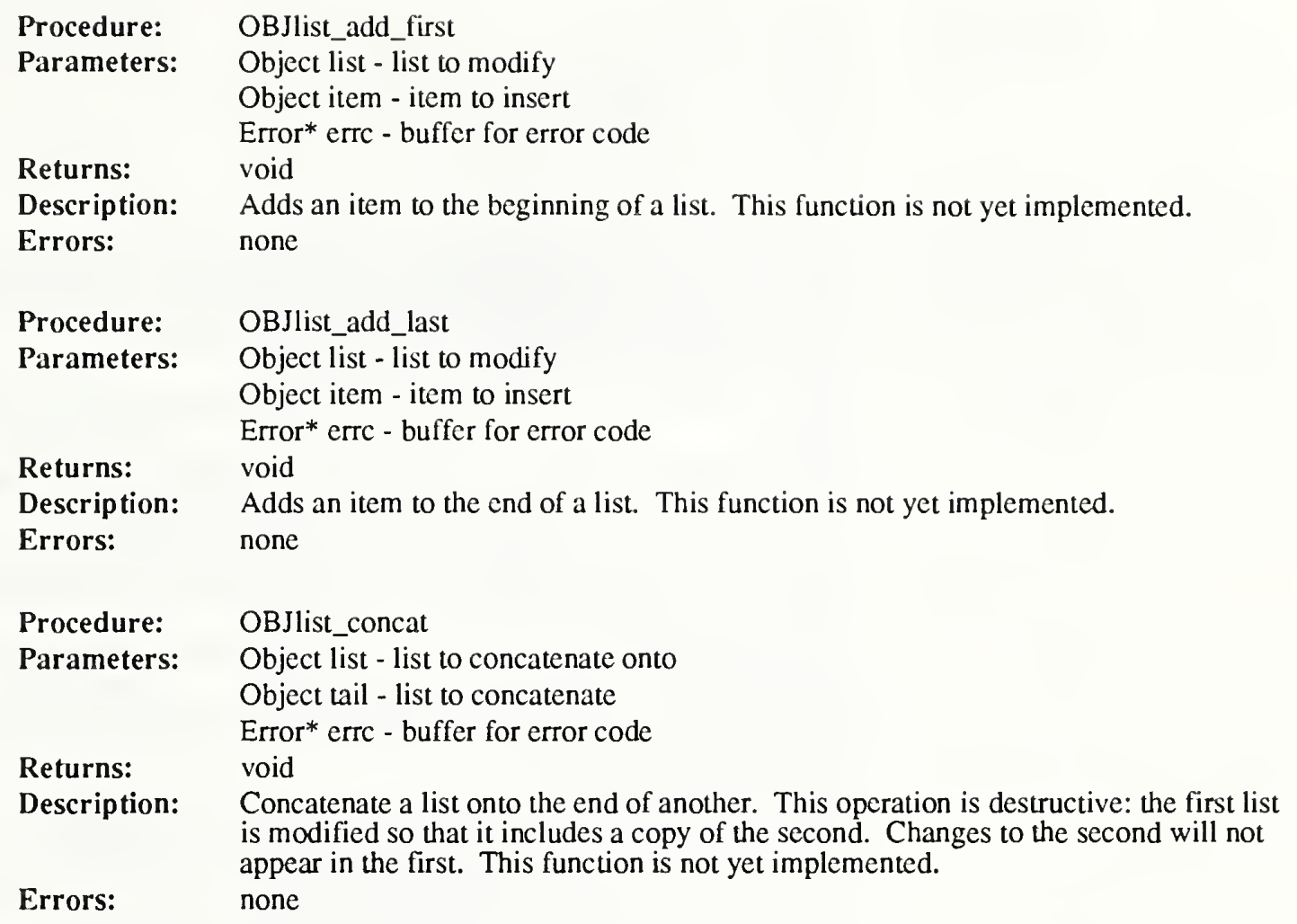


Procedure: OBJput_attributc

Parameters: $\quad$ Object object - objcct to modify

String attributeName - namc of attribute to store into

Objcct value - value to store into attribute

Error* errc - buffer for crror code

Returns: $\quad$ void

Requires: TYPEget_class(OBJget_type(objcct)) $==$ TYPE_ENTITY

Description: Stores a value into a named attribute of an entity instance. This call is the slower method for storing into an attribute. If the actual attribute record is available, for example from travcrsing the Entity's attribute list, use

OBJfast_put_attribute () instead.

Errors: $\quad$ ERROR_aggregate_expected - value given for an aggregate was not an aggregate

ERROR_array_expected - value given for an array was not an array

ERROR bag expected - value given for a bag was not a bag

ERROR entity expected - value given for an entity was not an entity

ERROR external expected - an external attribute was given an internal (embedded) entity as a value

ERROR inappropriate_entity - the entity given as a value was not of an expected class

ERROR_integer_expected - value given for an integer was not an integer ERROR internal_expected - an internal attribute was given an external entity reference as a value

ERROR_list_expected - value given for a list was not a list

ERROR logical expected - value given for a logical was not a logical

ERROR_number_expected - value given for a number was not a number

ERROR set expected - value given for a set was not a set

ERROR_string_expected - value given for a string was not a string

ERROR incompatible types - the value given is not of the expected type, in some way not covcred by any of the above messages

Procedure:

Parameters:

Returns:

Description:

Errors:

Procedure:

Parameters:

Returns:

Description:

Errors:

Procedure:

Parameters:

Returns:

Description:

Errors:
OBJput_linc_number

Object object - objcct to modify

int numbcr - line number for object

void

Sct an object's line numbcr.

none

OBJput_name

Object object - object to modify

String name - namc for object

void

Sets the name (identifier) of an object; normally, only entity instances which are not embedded arc named.

none

OBJput_user_data

Object object - object to modify

Generic valuc - user data value for object

Error* errc - buffer for error code

Generic - old value of user data field for this object

Stores a value into an object's user data field

none 


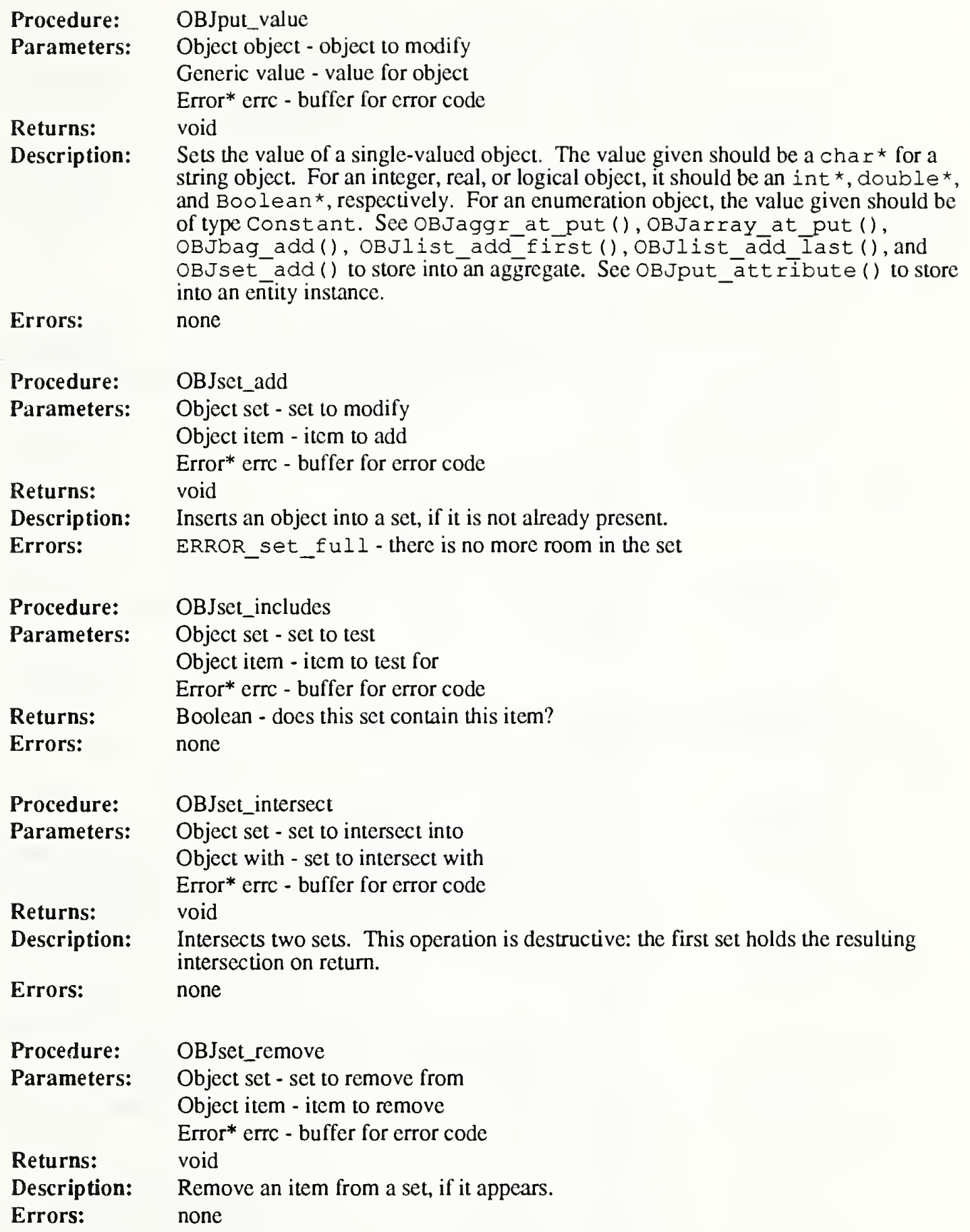


Procedure:

Parameters:

Returns:

Description:

Errors:

Procedure:

Parameters:

Returns:

Errors:

Procedure:

Parameters:

Returns:

Description:

Errors:

Procedure:

Parameters:

Returns:

Description:

Errors:
OBJset_remove_all

Object set - set to remove from

Object remove - set of items to remove

Error* errc - buffer for error code

void

Removes all items in a set from some other set. This is set subtraction. This operation is destructive: the first set holds the result on return.

none

\section{OBJset_subset}

Object set - set to test as superset

Object subset - set to test as subset

Error* errc - buffer for error code

Boolean - does the first set contain the second as a subset?

none

OBJset_unite

Object set - set to unite onto

Object unitee - set to unite with

Error* errc - buffer for error code

void

Forms the union of two sets. This operation is destructive: the first set holds the resulting union on return.

none

\section{OBJtype_cast}

Object object - object to be cast

Type type - type to cast to

Error* errc - buffer for error code

Object - the object, cast to the requested type

Converts an object to a new type, if possible. If the cast is successful ( ${ }^{*}$ rrc $==$

ERROR none), the original object should no longer be used. It is guaranteed to be valid only when an error is reported. This call does not report errors to stderr; it is the callers responsibility to check *errc and to call ERRORreport (*errc, (String) context) if it is not ERROR_none.

ERROR_aggregate_expected - value given for an aggregate was not an aggregate

ERROR_array_expected - value given for an array was not an array

ERROR_bag_expected - value given for a bag was not a bag

ERROR_entity_expected - value given for an entity was not an entity

ERROR inappropriate entity - the entity given as a value was not of an expected class

ERROR_integer_expected - value given for an integer was not an integer

ERROR_list_expected - value given for a list was not a list

ERROR_logical_expected - value given for a logical was not a logical

ERROR_number_expected - value given for a number was not a number

ERROR_set_expected - value given for a set was not a set

ERROR_string_expected - value given for a string was not a string

ERROR_incompatible_types - the value given is not of the expected type, in some way not covered by any of the above messages 
5.3 Product

Procedure: PRODadd_object

Parameters: Product product - product to modify

Object object - entity instance to add

Returns: $\quad$ void

Requires: TYPEget_class(OBJget_type(object)) $==$ TYPE_ENTITY

Description: Adds an entity instance to a product model. The instance is assumed already to have been added to the instance list of its class, since OBJcreate_entity () does this.

Errors: none

Procedure: PRODcreate

Parameters: String name - name for new product

Express model - conceptual schema in which to create product

Returns: $\quad$ Product - a new, empty product

Description: Creates an empty product within a particular conceptual schema.

Errors: none

Procedure: PRODget_conceptual_schema

Parameters: $\quad$ Product product - product to examine

Returns: $\quad$ Express - conceptual schema in which the product exists

Errors: none

Procedure: PRODget_contents

Parameters: $\quad$ Product product - product to examine

Returns: $\quad$ Linked_List - entity instances which make up the product

Description: Retrieves a list of the objects in a product model, in the order in which they were created.

Errors: none

Procedure: PRODget_name

Parameters: Product product - product to examine

Returns: $\quad$ String - the name of the product

Errors: none

Procedure: PRODget_named_object

Parameters: Product product - product to examine

String name - name of object to retrieve

Returns: Object - the named object

LDescription: Retrieves a named object from a STEP product model, if it is defined.

Errors: none

Procedure: PRODintialize

Parameters: -- none --

Returns: void

Description: Initializes the Product module. This is called by STEPinitialize0.

Errors: none 


\section{STEP Working Form Error Codes}

The Error module, which is used to manipulate these error codes, is described in [Clark90d]. All STEP Working Form error codes are defined in the Object module.

Error: $\quad$ ERROR_aggregate_expected

Severity: $\quad$ SEVERITY_ERROR

Meaning: $\quad$ A non-aggregate value was provided for an aggregate attribute

Format: $\quad \%$ s - attribute name

Error: $\quad$ ERROR_array_expected

Severity: $\quad$ SEVERITY_ERROR

Meaning: An aggregate of a specific non-array class was provided for an array attribute

Format: $\quad \%$ s - attribute name

Error: $\quad$ ERROR_bag_expected

Severity: $\quad$ SEVERITY_ERROR

Meaning: $\quad$ An aggregate of a specific non-bag class was provided for a bag attribute

Format: $\quad \%$ s - attribute name

Error: $\quad$ ERROR_bag_full

Severity: $\quad$ SEVERITY_WARNING

Meaning: $\quad$ An item was inserted into an already full bag

Format: $\quad$-- none --

Error: $\quad$ ERROR_cannot_instantiate

Severity: $\quad$ SEVERITY_ERROR

Meaning: $\quad$ An attempt was made to instantiate an uninstantiable type

Format: $\quad$ \%s - type name

Error: $\quad$ ERROR_entity_expected

Severity: $\quad$ SEVERITY_ERROR

Meaning: A non-entity Object was provided for an attribute having an entity type

Format: $\quad \%$ s - attribute name

Error: $\quad$ ERROR_external_expected

Severity: $\quad$ SEVERITY_WARNING

Meaning:

Format: $\quad$ \%s - attribute name

Error: $\quad$ ERROR_inappropriate_entity

Severity: $\quad$ SEVERITY_ERROR

Meaning: An entity of the wrong type was provided for an aturibute having an entity type

Format: $\quad$ \%s - attribute name

Error: $\quad$ ERROR_incompatible_types

Severity: $\quad$ SEVERITY_ERROR

Meaning: $\quad$ Some other type problem was encountered in specifying an attribute of some object.

Format: $\quad \%$ s-attribute name 


\begin{tabular}{|c|c|}
\hline Error: & ERROR_index_out_of_range \\
\hline Severity: & SEVERITY_WARNING \\
\hline Meaning: & An attempt was made to index an aggregate object outside of the legal bounds \\
\hline Format: & $\% \mathrm{~d}$ - index value \\
\hline Error: & ERROR_insufficient_attributes \\
\hline Severity: & SEVERITY_WARNING \\
\hline Meaning: & Too few attribute values were provided for a particular entity instantiation \\
\hline Format: & $\% s$ - entity instance identifier \\
\hline Error: & ERROR_integer_expected \\
\hline Severity: & SEVERITY_ERROR \\
\hline Meaning: & A non-integer value was provided for an integer attribute \\
\hline Format: & $\% s$ - attribute name \\
\hline Error: & ERROR_internal_expected \\
\hline Severity: & SEVERITY WARNING \\
\hline Meaning: & $\begin{array}{l}\text { An non-embedded (external) entity was provided for an attribute with "internal" } \\
\text { reference class }\end{array}$ \\
\hline Format: & $\%$ s - attribute name \\
\hline Error: & ERROR_list_expected \\
\hline Severity: & SEVERITY_ERROR \\
\hline Meaning: & An aggregate of a specific non-list class was provided for a list attribute \\
\hline Format: & $\% s$ - attribute name \\
\hline Error: & ERROR_logical_expected \\
\hline Severity: & SEVERITY_ERROR \\
\hline Meaning: & A non-logical value was provided for a logical attribute \\
\hline Format: & $\% s$ - attribute name \\
\hline Error: & ERROR_number_expected \\
\hline Severity: & SEVERITY_ERROR \\
\hline Meaning: & A non-numeric value was provided for a numeric attribute \\
\hline Format: & $\%$ s - attribute name \\
\hline Error: & ERROR_set_duplicate_entry \\
\hline Severity: & SEVERITY_ERROR \\
\hline Meaning: & A duplicate entry was added to a set \\
\hline Format: & -- none -- \\
\hline Error: & ERROR_set_expected \\
\hline Severity: & SEVERITY_ERROR \\
\hline Meaning: & An aggregate of a specific non-set class was provided for a set attribute \\
\hline Format: & $\% s$ - attribute name \\
\hline Error: & ERROR_set_full \\
\hline Severity: & SEVERITY_WARNING \\
\hline Meaning: & An item was inserted into an already full set \\
\hline Format: & -- none -- \\
\hline
\end{tabular}




$\begin{array}{ll}\text { Error: } & \text { ERROR_string_expected } \\ \text { Severity: } & \text { SEVERITY_ERROR } \\ \text { Meaning: } & \text { A non-string Object was provided for a string attribute } \\ \text { Format: } & \text { \%s - attribute name } \\ & \\ \text { Error: } & \text { ERROR_too_many_attributes } \\ \text { Severity: } & \text { SEVERITY_WARNING } \\ \text { Meaning: } & \text { Too many attribute values were provided for a particular entity instantiation } \\ \text { Format: } & \text { \%s - entity instance identifier } \\ & \\ \text { Error: } & \text { ERROR_undefined_reference } \\ \text { Severity: } & \text { SEVERITY_ERROR } \\ \text { Meaning: } & \text { A reference was made to an unknown entity instance identifier } \\ \text { Format: } & \text { \%s - entity instance identifier } \\ & \\ \text { Error: } & \text { ERROR_unknown_entity } \\ \text { Severity: } & \text { SEVERITY_ERROR } \\ \text { Meaning: } & \text { A reference was made to an unknown entity class (type) } \\ \text { Format: } & \text { \%s - entity class name }\end{array}$




\section{A References}

[Altemueller88] Altemueller, J., The STEP File Structure, ISO TC184/SC4/WG1 Document N279, September, 1988

[ANSI89] American National Standards Institute, Programming Language C, Document ANSI X3.159-1989

[Clark90a] Clark, S. N., An Introduction to The NIST PDES Toolkit, NISTIR 4336, National Institute of Standards and Technology, Gaithersburg, MD, May 1990

[Clark90b] Clark, S.N., Fed-X: The NIST Express Translator, NISTIR, National Institute of Standards and Technology, Gaithersburg, MD, forthcoming

[Clark90c] Clark, S.N., The NIST Working Form for STEP, NISTIR 4351, National Institute of Standards and Technology, Gaithersburg, MD, June 1990

[Clark90d] Clark, S.N., The NIST PDES Toolkit: Technical Fundamentals, NISTIR 4335, National Institute of Standards and Technology, Gaithersburg, MD, May 1990

[Clark90e] Clark, S.N., NIST Express Working Form Programmer's Reference, NISTIR, National Institute of Standards and Technology, Gaithersburg, MD, forthcoming

[Schenck89] Schenck, D., ed., Information Modeling Language Express: Language Reference Manual, ISO TC184/SC4/WG1 Document N362, May 1989

[Smith88] Smith, B., and G. Rinaudot, eds., Product Data Exchange Specification First Working Draft, NISTIR 88-4004, National Institute of Standards and Technology, Gaithersburg, MD, December 1988 



\section{MAIL TO:}

NATIONAL - National Institute of Standards and Technology

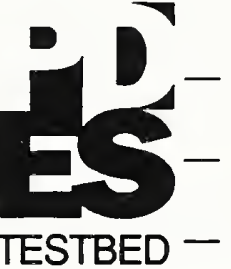
Gaithersburg MD., 20899

Metrology Building, Rm-A127

Attn: Secretary National PDES Testbed (301) 975-3508

\section{Please send the following documents and/or software:}

Clark, S.N., An Introduction to The NIST PDES Toolkit

Clark, S.N., The NIST PDES Toolkit: Technical Fundamentals

Clark, S.N., Fed-X: The NIST Express Translator

Clark, S.N., The NIST Working Form for STEP

Clark, S.N., NIST Express Working Form Programmer's Reference

Clark, S.N., NIST STEP Working Form Programmer's Reference,

Clark, S.N., QDES User's Guide

Clark, S.N., QDES Administrative Guide

Morris, K.C., Translating Express to SQL: A User's Guide

Nickerson, D., The NIST SQL Database Loader: STEP Working Form to $\underline{S Q L}$

Strouse, K., McLay, M., The PDES Testbed User's Guide

OTHER (PLEASE SPECIFY)

These documents and corresponding software will be available from NTIS in the future. When available, the NTIS ordering information will be forthcoming. 



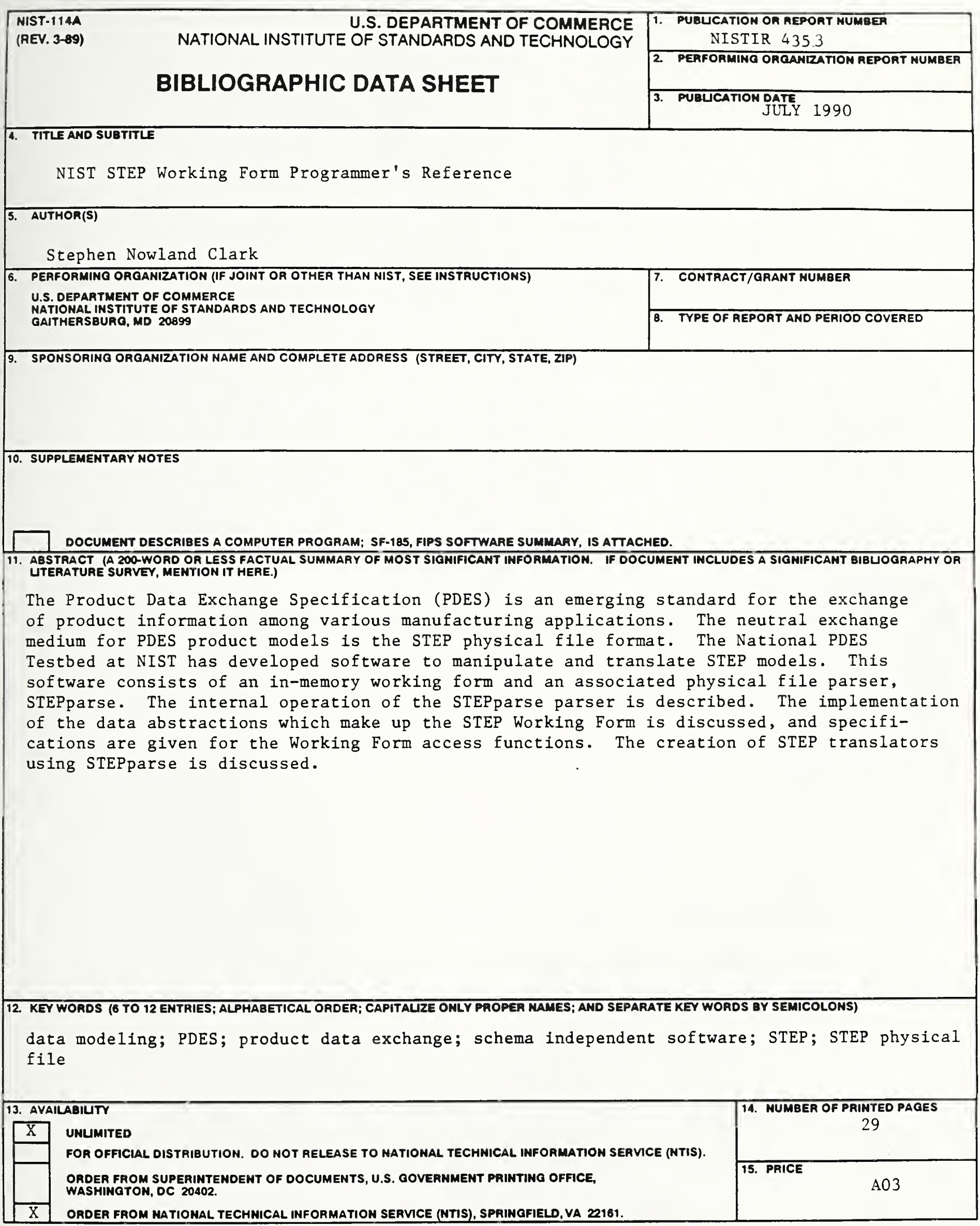



